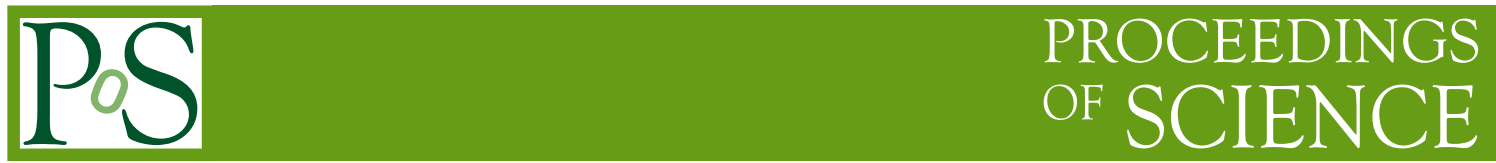

\title{
$\boldsymbol{A b}$ initio theory of light nuclei and $N N$ interaction of JISP type
}

\author{
Andrey M. Shirokov* \\ Skobeltsyn Institute of Nuclear Physics, Moscow State University, Moscow, Russia \\ Department of Physics and Astronomy, Iowa State University, Ames IA, USA \\ Pacific National University, Khabarovsk, Russia \\ E-mail: shirokovenucl-th.sinp.msu.ru
}

I discuss ab initio studies of light nuclei in large-scale No-Core Shell Model calculations on supercomputers with extrapolations to the infinite model space (No-Core Full Configuration approach). The convergence at the end of $p$ shell and beginning of the $s d$ shell can be achieved if only reasonable soft enough $N N$ interactions are used. In particular, good predictions are obtained with a realistic JISP16 $N N$ interaction obtained in $J$-matrix inverse scattering approach and fitted to reproduce light nuclei observables without three-nucleon forces. We discuss some recent results obtained with this $N N$ interaction.

The XXI International Workshop High Energy Physics and Quantum Field Theory,

June 23 - June 30, 2013

Saint Petersburg Area, Russia

\footnotetext{
* Speaker.
} 


\section{INTRODUCTION}

One of the mainstreams of modern nuclear theory is an $a b$ initio description of nuclei, i. e. model-free calculations of many-nucleon systems using supercomputers. A rapid development of $a b$ initio methods for solving finite nuclei has opened a range of nuclear phenomena that can be evaluated to high precision using realistic nucleon-nucleon interactions. Nowadays, due to increased computing power and novel techniques, ab initio approaches like the No-Core Shell Model (NCSM) [1], the Green's Function Monte Carlo [2] and the Coupled-Cluster Theory [3] are able to reproduce properties of a large number of atomic nuclei with mass up to $A=16$ and can be extended for heavier nuclei. Recently a new ab initio method, the No-Core Full Configuration (NCFC) approach [ $₫$ ], was introduced. NCFC is based on extrapolation of the NCSM results in successive basis spaces to the infinite basis space limit. This makes it possible to obtain basis space independent predictions for binding energies and to evaluate their numerical uncertainties. We concentrate the discussion here on the NCFC approach and on some new results obtained with it.

The $a b$ initio methods require a reliable realistic strong interaction providing an accurate description of $N N$ scattering data and high-quality predictions for binding energies, spectra and other observables in light nuclei. A number of meson-exchange potentials sometimes supplemented with phenomenological terms to achieve high accuracy in fitting $N N$ data (CD-Bonn [5], Nijmegen [6], Argonne [7]) have been developed that should be used together with modern $N N N$ forces (Urbana[8, 9], Illinois [10], Tucson-Melbourne [11]-13]]) to reproduce properties of manybody nuclear systems. On the other hand, one sees the emergence of realistic $N N$ and $N N N$ interactions with ties to QCD via Chiral Perturbation Theory [14-17].

Three-nucleon forces require a significant increase of computational resources needed to diagonalize a many-body Hamiltonian matrix since the $N N N$ interaction increases the number of non-zero matrix elements approximately by a factor of 30 in the case of $p$-shell nuclei. As a result, one needs to restrict the basis space in many-body calculations when $N N N$ forces are involved that makes the predictions less reliable. Ab initio many-body studies benefit from the use of recently developed purely two-nucleon interactions of INOY (Inside Nonlocal Outside Yukawa) [18, 19] and JISP ( $J$-matrix Inverse Scattering Potential) [20-23] types fitted not only to the $N N$ data but also to binding energies of $A=3$ and heavier nuclei. At the fundamental level, these $N N$ interactions are supported by the work of Polyzou and Glöckle [24] who demonstrated that a realistic $N N$ interaction is equivalent at the $A=3$ level to some $N N+N N N$ interaction where the new $N N$ force is related to the initial one through a phase-equivalent transformation (PET). It seems reasonable then to exploit this freedom and work to minimize the need for the explicit introduction of threeand higher-body forces. Endeavors along these lines have resulted in the design of INOY and JISP strong interaction models.

Conventional realistic meson-exchange $N N$ interactions [5]-7] and $N N$ interactions obtained in Chiral Perturbation Theory [16] do not provide a fast convergence of many-body calculations. To improve the convergence, these $N N$ interactions are usually softened either using the OkuboLee-Suzuki (OLS) [25, 26] method reviewed in detail in Ref. [27] or the Similarity Renormalization Group (SRG) technique [28, 29]. Unfortunately, the convergence of many-body calculations with OLS-softened $N N$ interactions is not monotonic as the basis space is enlarged, and hence the respective results cannot be extrapolated to the infinite basis space. The SRG softening of $N N$ 
interaction induces $N N N$ forces.

We review briefly the results obtained with JISP $N N$ interactions. The JISP $N N$ interaction provides a fast convergence of NCSM calculations, it does not require the use of $N N N$ forces and is fitted in NCSM and NCFC studies to the properties of light nuclei and is developing together with the progress in these ab initio approaches.

\section{JISP16 $N N$ INTERACTION AND NCFC APPROACH}

The $J$-matrix inverse scattering approach was suggested in Ref. [30]. It was further developed and used to design a high-quality JISP NN interaction in Ref. [20]. A nonlocal interaction obtained in this approach is in the form of a matrix in the oscillator basis in each of $N N$ partial waves. To reproduce scattering data in a wider energy range, one needs to increase the size of the potential matrix and/or the $\hbar \Omega$ parameter of the oscillator basis. From the point of view of shell model applications, it is desirable however to reduce the size of potential matrices and to use $\hbar \Omega$ values in the range of few tens of $\mathrm{MeV}$. A compromise solution is to use $\hbar \Omega=40 \mathrm{MeV}$ with $N_{\max }=9$ truncation of potential matrices, i. e., the JISP $N N$ interaction matrices include all relative $N N$ motion oscillator states with excitation quanta up to 8 or 9 depending on parity. In other words, we use potential matrices of the rank $r=5$ in $s$ and $p$ partial waves, $r=4$ matrices in $d$ and $f$ partial waves, etc. In case of coupled waves, the rank of the potential matrix is a sum of the respective ranks, e.g., the rank of the coupled $s d$-wave matrix is $r=5+4=9$. The $N_{\max }=9$ truncated JISP interaction with $\hbar \Omega=40 \mathrm{MeV}$ provides an excellent description of $N N$ scattering data with $\chi^{2} /$ datum $=1.03$ for the $1992 n p$ data base (2514 data), and 1.05 for the $1999 n p$ data base (3058 data) [31].

PETs originating from unitary transformations of the oscillator basis proposed in Refs. [32, 33], give rise to ambiguity of the interaction obtained in the $J$-matrix inverse scattering approach. This ambiguity is eliminated at the first stage by postulating the simplest tridiagonal form of the $N N$ interaction in uncoupled and quasi-tridiagonal form in coupled $N N$ partial waves [20]. At the next stage, PETs are used to fit the JISP interaction to various nuclear properties. First of all, the $s d$ component of the $N N$ interaction is modified with the help of PETs to reproduce the deuteron quadrupole moment $Q$ and rms radius without violating the excellent description of scattering data. It is worth noting here that the deuteron binding energy $E_{d}$ and asymptotic normalization constants are used as an input in the inverse scattering approach and are not affected by PETs.

After that we employ PETs in other $N N$ partial waves attempting to improve the description of binding energies and spectra of light nuclei in NCSM calculations. Following this ab exitu route, the JISP6 $N N$ interaction fitted to properties of nuclei with masses $A \leq 6$, was proposed in Refs. [21, 22]. It was found out later that JISP6 strongly overbinds nuclei with $A \geq 10$. Therefore a new fit of PET parameters was performed that resulted in the JISP16 NN interaction [23, 34] fitted to binding energies of nuclei with masses up through $A \leq 16$.

The JISP16 $N N$ interaction provides a good description of binding energies, spectra and other properties of $s$ and $p$ shell nuclei. It was used in a number of papers of various groups (see, e.g., Refs. [ [ 23, 35-49]) and was shown to be one of the best if not the best as compared to other modern models of the realistic strong interaction from the point of view of description of observables in light nuclei. It is worth noting that JISP16 provides better convergence of ab initio 
calculations than other realistic $N N$ interactions and avoids the need to use three-nucleon forces. As a result, the JISP16 predictions for light nuclei are more reliable than that of other realistic models of $N N$ interactions. With modern supercomputer facilities, we can obtain converged or nearly converged energies of nuclei with mass $A \leq 6$. For calculations of heavier nuclear systems, we proposed recently a NCFC approach [ $₫$.

It was found [4] that binding energies of many light nuclei represent an exponential convergence pattern in the excitation oscillator quanta $N_{\max }$ characterizing the basis space of the NCSM. Therefore, we fit the set of ground state energies obtained with each fixed $\hbar \Omega$ value using the relation

$$
E_{\mathrm{gs}}\left(N_{\max }\right)=A \exp \left(-c N_{\max }\right)+E_{\mathrm{gs}}(\infty),
$$

where fitting parameters $A$ and $c$ depend on the $\hbar \Omega$ value and $E_{\mathrm{gs}}(\infty)$ is the extrapolated ground state energy in the infinite basis space. We have suggested [ $₫$ ] method of evaluating of uncertainty of theoretical prediction for the extrapolated energy $E_{\mathrm{gs}}(\infty)$.

An exciting recent result obtained with JISP16 NN interaction and NCFC method, is an $a b$ initio prediction [46] of properties of the exotic extreme proton-excess nucleus ${ }^{14} \mathrm{~F}$. The first experimental results regarding this isotope became available recently from Cyclotron Institute at Texas A\&M University [50]. The largest calculations were performed in the NCSM basis space with $N_{\max }=8$, which for this nucleus contains 1990061078 basis states with natural (negative) parity. The determination of the lowest ten to fifteen eigenstates of the sparse Hamiltonian matrix, for each oscillator parameter requires 2 to 3 hours on 30504 quad-core compute nodes at the Jaguar supercomputer at ORNL. Our prediction for the ${ }^{14} \mathrm{~F}$ binding energy of $72 \pm 4 \mathrm{MeV}$ was nicely confirmed by a later experiment [50] where a value of $74.00 \mathrm{MeV}$ was reported.

Several excited states in ${ }^{14} \mathrm{~F}$ were observed experimentally [50]. They are compared with our predictions [46] in Fig. 1. The experiment [50] is seen to confirm our predictions for the ${ }^{14} \mathrm{~F}$ spectrum obtained before the first observation of this nucleus. These results provide a strong support to our ab initio approach based on NCSM calculations, NCFC extrapolations and the use of realistic JISP16 $N N$ interaction. The ab initio results are seen from Fig. 1 to reproduce the experiment much better than conventional shell model calculations with an inert core and phenomenological effective interactions WPB and MK.

The use of newest supercomputers and NCFC technique revealed some deficiency of the JISP16 NN interaction. In particular, it overbinds nuclei at the closure of the $p$ shell like ${ }^{16} \mathrm{O}$.

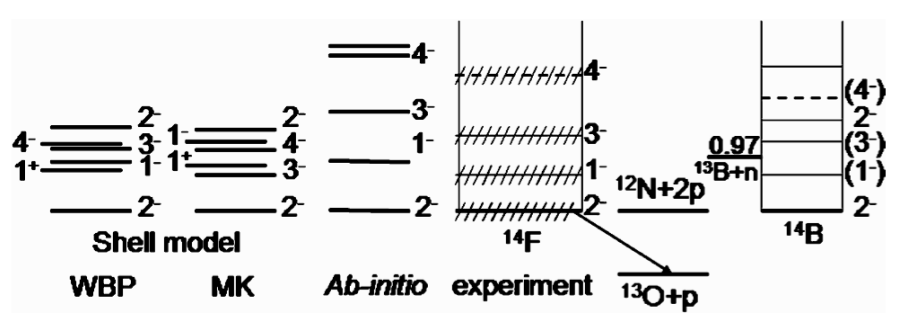

Figure 1: The ${ }^{14} \mathrm{~F}$ spectrum: shell model calculations with WPB and MK interactions [50] and our predictions [46] (Ab-initio) in comparison with experimental data of Ref. [50] (experiment) and that of the mirror ${ }^{14} \mathrm{~B}$ nucleus [51]. 
A new version of this interaction JISP16 2010 was recently suggested [45]. The JISP16 $2010 N N$ interaction improves essentially the description of binding energies of nuclei with the mass $A \leq 16$.

This work was supported in part by the Ministry of Education and Science of the Russian Federation through the contract 14.V37.21.1297 and by US DOE Grants DE-FC02-09ER41582 and DE-FG02-87ER40371. Computational resources were provided by DOE (supercomputers Hopper and Jaguar) and by Moscow State University (supercomputer Lomonosov).

\section{References}

[1] P. Navrátil, J. P. Vary and B. R. Barrett, Phys. Rev. Lett. 84 (2000) 5728; Phys. Rev. C 62 (2000) 054311.

[2] S. Pieper and R. B. Wiringa, Annu. Rev. Nucl. Part. Sci. 51 (2001) 53.

[3] K. Kowalski, D. J. Dean, M. Hjorth-Jensen, T. Papenbrock and P. Piecuch, Phys. Rev. Lett. 92 (2004) 132501.

[4] P. Maris, J. P. Vary, A. M. Shirokov, Phys. Rev. C 79 (2009) 014308.

[5] R. Machleidt, Phys. Rev. C 63 (2001) 024001.

[6] V. G. J. Stoks, R. A. M. Klomp, C.P.F. Terheggen and J. J. de Swart, Phys. Rev. C 49 (1994) 2950.

[7] R. B. Wiringa, V. G. J. Stoks and R. Schiavilla, Phys. Rev. C 51 (1995) 38.

[8] J. Carlson, V. R. Pandharipande and R. B. Wiringa, Nucl. Phys. A 401 (1983) 59.

[9] B. S. Pudliner, V. R. Pandharipande, J. Carlson, S. C. Pieper and R. B. Wiringa, Phys. Rev. C 56 (1997) 1720.

[10] S. C. Pieper, V. R. Pandharipande, R. B. Wiringa and J. Carlson, Phys. Rev. C 64 (2001) 014001.

[11] S. A. Coon, M. D. Scadron, P. C. McNamee, B. R. Barrett, D. W. E. Blatt and B. H. J. McKellar, Nucl. Phys. A 317 (1979) 242.

[12] J. L. Friar, D. Hüber and U. van Kolck, Phys. Rev. C 59 (1999) 53.

[13] D. Hüber, J. L. Friar, A. Nogga, H. Witała and U. van Kolck, Few-Body Syst. 30 (2001) 95.

[14] P. F. Bedaque, H.-W. Hammer and U. van Kolck, Phys. Rev. Lett. 82 (1999) 463.

[15] E. Epelbaum, A. Nogga, W. Glö ckle, H. Kamada, U.-G. Meißner and H. Witała, Phys. Rev. C 66 (2002) 064001.

[16] D. R. Entem and R. Machleidt, Phys. Lett. B 524 ( 2002) 93; Phys. Rev. C 68 (2003) 041001(R).

[17] P. Navrátil, V. G. Gueorguiev, J. P. Vary, A. Nogga and W. E. Ormand, Phys. Rev. Lett. 99 (2007) 042501.

[18] P. Doleschall, Phys. Rev. C 69 (2004) 054001.

[19] P. Doleschall, I. Borbé ly, Z. Papp and W. Plessas, Phys. Rev. C 67 (2003) 064005.

[20] A. M. Shirokov, A. I. Mazur, S. A. Zaytsev, J. P. Vary and T. A. Weber, Phys. Rev. C 70 (2004) 044005 .

[21] A. M. Shirokov, J. P. Vary, A. I. Mazur, S. A. Zaytsev and T. A. Weber, Phys. Lett. B 621 (2005) 96. 
[22] A. M. Shirokov, J. P. Vary, A. I. Mazur, S. A. Zaytsev and T. A. Weber, J. Phys. G 31 (2005) S1283.

[23] A. M. Shirokov, J. P. Vary, A. I. Mazur and T. A. Weber, Phys. Lett. B 644 (2007) 33.

[24] W. N. Polyzou and W. Glöckle, Few-Body Syst. 9 (1990) 97.

[25] S. Okubo, Progr. Theor. Phys. 12 (1954) 603.

[26] K. Suzuki and S. Y. Lee, Progr. Theor. Phys. 64 (1980) 2091.

[27] B. R. Barrett, P. Navratil and J. P. Vary, Progr. Part. Nucl. Phys. 69, 131 (2013).

[28] S. D. Glazek and K. G. Wilson, Phys. Rev. D 48 (1993) 5863.

[29] F. Wegner, Ann. Phys. (NY) 506 (1994) 77.

[30] S. A. Zaytsev, Theor. Math. Phys. 115 (1998) 575.

[31] R. Machleidt, private communication (2006).

[32] Yu. A. Lurie and A. M. Shirokov, Bull. Rus. Acad. Sci. Phys. 61 (1997) 1665.

[33] Yu. A. Lurie and A. M. Shirokov, Ann. Phys. (NY) 312 (2004) 284.

[34] A Fortran code generating the JISP16 interaction matrix elements is available at http://nuclear.physics.iastate.edu.

[35] N. Barnea, W. Leidemann and G. Orlandini, Phys. Rev. C 74 (2006) 034003.

[36] W. Leidemann, Nucl. Phys. A 790 (2007) 24.

[37] G. Orlandini, S. Bacca, N. Barnea and W. Leidemann, Nucl. Phys. A 790 (2007) 368.

[38] I. Šlaus. Nucl. Phys. A 790 (2007) 199.

[39] T. Dytrych, K. D. Sviratcheva, C. Bahri, J. P. Draayer and J. P. Vary, Phys. Rev. Lett. 98 (2007) 162503.

[40] A. M. Shirokov, J. P. Vary, A. I. Mazur and T. A. Weber, Phys. At. Nucl. 71 (2008) 1232.

[41] A. M. Shirokov, J. P. Vary and P. Maris, in proceedings of 27th Int. Workshop Nucl. Theory, Rila Mountains, Bulgaria, 23-28 June, 2008 (Ed. S. Dimitrova). Bulgarian Acad. Sci., Sofia, 2008, p. 205; arXiv:0810.1014 [nucl-th] (2008).

[42] J. P. Vary, P. Maris and A. Shirokov, Int. J. Mod. Phys. E 17, Suppl. 1 (2008) 109.

[43] A. M. Shirokov, A. I. Mazur, J. P. Vary and E. A. Mazur, Phys. Rev. C 79 (2009) 014610.

[44] S. Vaintraub, N. Barnea and D. Gazit, Phys. Rev. C 79 (2009) 065501.

[45] A. M. Shirokov, V. A. Kulikov, P. Maris, A. I. Mazur, E. A. Mazur and J. P. Vary, EPJ Web of Conf. 3 (2010) 05015.

[46] P. Maris, A. M. Shirokov, J. P. Vary, Phys. Rev. C 81 (2010) 021301(R).

[47] G. Orlandini, N. Barnea and W. Leidemann, J. Phys. Conf. Ser. 312 (2011) 092049.

[48] K. D. Launey, T. Dytrych and Jerry P. Draayer, Phys. Rev. C 85 (2012) 044003.

[49] T. Dytrych, K. D. Launey, J. P. Draayer, P. Maris, J. P. Vary, E. Saule, U. Catalyurek, M. Sosonkina, D. Langr and M. A. Caprio, Phys. Rev. Lett. 111 (2013) 25250.

[50] V. Z. Goldberg, B. T. Roeder, G. V. Rogachev, G. G. Chubarian, E. D. Johnson, C. Fu, A. A. Alharbi, M. L. Avila, A. Banu, M. McCleskey, J. P. Mitchell, E. Simmons, G. Tabacaru, L. Trache and R. E. Tribble, Phys. Lett. B 692 (2010) 307.

[51] F. Ajzenberg-Selove, Nucl. Phys. A 523 (1991) 1. 